\title{
Editorial
}

\section{A NICE year for CBT and a CBT year for NICE}

This is going to be an interesting year for CBT. The National Institute of Clinical Excellence (NICE) unveils a series of treatment guidelines this year. These guidelines are intended to support good practice in clinical settings; implicitly, they also seek to discourage ineffective or bad practice. It is becoming evident that CBT is likely to be judged as a first line treatment (or the first line treatment) in a range of anxiety disorders, depression and eating disorders. Other issues which will be tackled include the lack of evidence for just about all other psychological treatment approaches, that CBT should be readily and rapidly available where it is needed and the need for quality control linked with (but not confined to) proper training and supervision of therapists. Early identification of clinical difficulties and a better research understanding of the extent and limitations of current psychological and pharmacological treatments are also likely to be advocated. These are all issues which greatly concern the Association and this journal, and as editor I look forward to receiving empirically based submissions addressing these topics.

What the guidelines will not do, because it is explicitly beyond their scope, is address the appalling resource problem which confronts mental health services and primary care. Only a tiny fraction of people who presently conduct psychological treatment have been adequately trained in CBT. Is this being rectified by changes in current training priorities? Sadly, there is no sign of any concerted attempt to increase training in empirically grounded psychotherapy. The vast majority of people completing psychotherapy training over the next three years will be well qualified to offer treatment which the NICE guidelines do not recommend on the basis that there is not even low grade evidence for their effectiveness. Ineffective psychological treatment will continue to be offered to those with mental health problems simply because that is all that is readily available and there is little prospect of this changing in the foreseeable future. Most patients will probably be offered medication as the first line of treatment simply on the basis of availability. A radical reconsideration of service delivery and training models is long overdue. However, it is hard to detect even a flicker of awareness of these problems at the policy level. Organisations such as the United Kingdom Council for Psychotherapy, the British Psychological Society and the British Confederation of Psychotherapists might, to the outsider, appear preoccupied with guild issues at the expense of effective interventions for people with mental health problems.

It seems likely that a previously neglected factor will begin to become more important. The role of the service user, patient or consumer is set to become more important. Within medicine the notions of "Shared Decision Making" and "Evidence Based Patient Choice" are increasingly recognised as important factors and are beginning to be applied in mental health. It is appropriate that the service user would want to know what the range of treatment options are and to know that the treatment decision made is based on a clear understanding of the pros and cons of each option and how these match their personal situation and values. This notion is indeed part of the impetus for the work of NICE, and a key part of the output of the guidelines 
groups will be public information leaflets. People seeking a service such as psychotherapy are usually interested in how likely it is to actually help them and how long that will take.

Finally, you will notice in this issue and hereafter that the "Clinical Section" is no longer present. Since the introduction of "Brief Clinical Reports", the status of this section has been ambiguous, and we have decided to remove it. This will have no impact on journal policy, and we will continue to publish papers with a predominantly clinical emphasis.

Paul Salkovskis, Editor 